\title{
Iterative Mesh Generation for FE-Computations on Free Form Surfaces
}

\author{
Leif Kobbelt* \\ Torsten Hesse ${ }^{\dagger}$ \\ Hartmut Prautzsch ${ }^{\ddagger}$ \\ Karl Schweizerhof ${ }^{\dagger}$
}

May 30, 1996

\begin{abstract}
We present an interpolatory subdivision scheme to generate adaptiely refined quadrilateral meshes which approximate a smooth surface of arbitrary topology. The described method significantly differs from classical mesh generation techniques based on spline surfaces or implicit representations since no explicit description of the limit surface is used. Instead, simple affine combinations are applied to compute new vertices if a face of the net is split. These rules are designed to guarantee asymptotic smoothness, i.e., the sequence of refined nets converges to a smooth limit surface. Subdivision techniques are useful mainly in applications where a given quadrilateral net is a coarse approximation of a surface and points on a refined grid have to be estimated. To evaluate our approach, we show examples for FE-computations on surfaces generated by this algorithm.
\end{abstract}

\section{Introduction}

To perform numerical computations on surfaces, one usually starts by discretizing the problem, i.e., a smooth manifold is approximated by a triangular or quadrilateral net. The faces of such nets can be filled by linear or bilinear elements respectively. The size of the faces controls the

\footnotetext{
${ }^{*}$ Computer Sciences Department, University of Wisconsin - Madison, 1210 West Dayton Street, Madison, WI 53706-1685, USA, kobbelt@cs.wisc.edu

$\dagger$ Universität Karlsruhe, Institut für Mechanik, 76128 Karlsruhe, Germany

‡Universität Karlsruhe, Institut für Betriebs- und Dialogsysteme, 76128 Karlsruhe, Germany
}

quality of the approximation and thus the reliability of the computation. The trade-off between computational complexity and error tolerance makes it necessary to adapt the grid size of the discretization during the computation. Usually one starts with a rather coarse approximation and then iteratively refines the net until the required precision is obtained.

In many applications, the underlying surface itself is given by a set of data points, e.g., scanned from a real object. In a pre-processing step, the topology has to be determined, i.e., the points have to be connected to build an initial mesh (reverse engineering). Then one can apply approximation or interpolation techniques to construct a smooth surface fitting the data. However, to perform finite element computations, this surface again has to be sampled on some (finer) grid thus returning to a discrete representation (cf. Fig. 1). The standard methods describe such constructions usually by means of spline surfaces [HL93] or implicit representations [BCX94a, BCX94b].

If we are interested only in the meshes themselves, it makes sense to avoid the explicit construction of an interpolating surface and to derive the refined net directly from the given one. This technique is called interpolatory subdivision or refinement. A refinement scheme is completely defined by a set of rules how to compute new vertices. These rules are usually simple affine combinations of points from the unrefined net. If the coefficients of these combinations satisfy certain conditions [Rei95, Pra95] then the iterative refinement generates a sequence of finer and finer nets which converge to a tangent-plane continuous surface.

The justification for this approach is that one 


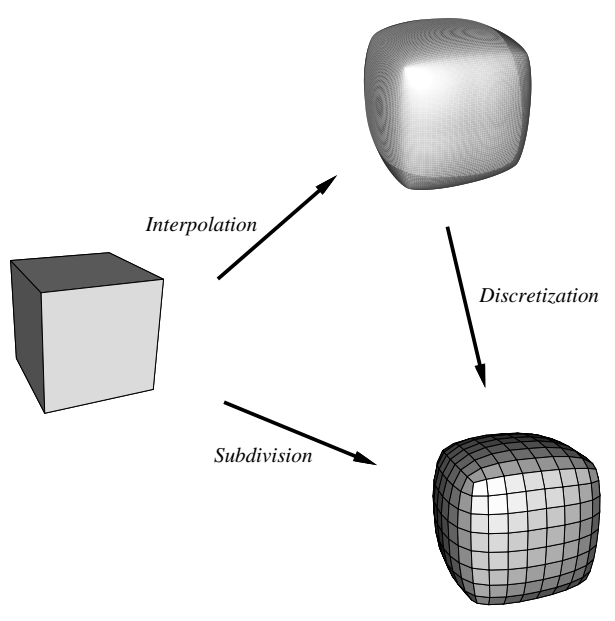

Figure 1: Generation of interpolating surfaces

avoids the mathematically involved construction of smooth parametric surfaces but uses the initial mesh obtained during the reverse engineering process directly or after some regularization. If a refinement of the mesh is needed to achieve a prescribed tolerance, the net is refined by simple fixed rules to compute the locations of the new points.

In the literature the term subdivision usually refers to a more general class of algorithms which generate sequences of polyhedral nets [DS78, CC78, Loo87, Loo94, Kob96]. Most of these schemes are derived from knot-insertion algorithms of piecewise polynomial tensor-product splines. In this paper we restrict ourselves to the special case of interpolatory subdivision where the net refinement is achieved by inserting new vertices and keeping the existing vertices unchanged.

A fairly well-known algorithm of this subclass is the butterfly-algorithm of [DGL90, ZSS96]. However, this algorithm operates on triangular nets only. For our application of FEcomputations on surfaces, quadrilateral (bilinear) elements turn out to be more appropriate since, especially for varying gradients, the geometry is approximated better with the same number of degrees of freedom and locking problems are overcome [BD86, SF89a, SF89b].

In the following, we first describe an interpolatory subdivision scheme for closed quadrilateral nets which, in the limit, generates smooth $\left(C^{1}\right)$ surfaces of arbitrary topological type. A slight modification of the scheme allows us to refine open nets in which case smooth boundary curves interpolate the given boundary polygon.

Since the computation of new vertices only requires local information from the preceeding refinement level, local refinement strategies can be applied. However, to keep the mesh $C^{0}$ consistent the locally refined net has to remain balanced, i.e., the refinement level of two faces having at least one vertex in common, must not differ by more than 1 . We refer to wellknown techniques to handle balanced quadrilateral meshes [PSB90].

We derive the approximation order, i.e., the factor by which the approximation to a real surface $S$ is improved if we start the iterative refinement on a finer initial mesh (with points lying exactly on $S$ ). Further, we compare the results of FE-computations on meshes generated by our refinement scheme to results obtained by other mesh refinement techniques. The experimental results demonstrate the practical usefulness of the scheme.

\section{Subdivision Rules}

The following description is intended to be as concise as possible. A more detailed presentation can be found in [Kob95, Kob96].

Uniform refinement of a quadrilateral mesh means to compute one new vertex for each edge and one for each face. We call these new vertices edge-vertices $(E)$ and face-vertices $(F)$ respectively (cf. Fig. 2). For both types of new vertices, we define a separate refinement rule to determine their position.

We label the vertices as shown in Fig. 3. Let e be the vertex associated with the edge $\overline{\mathbf{p}_{0} \mathbf{q}_{0}}$ then

$$
\mathbf{e}:=\frac{4+w}{8}\left(\mathbf{p}_{0}+\mathbf{q}_{0}\right)-\frac{w}{8}\left(\mathbf{p}^{*}+\mathbf{q}^{*}\right),
$$

where $\mathbf{p}^{*}, \mathbf{q}^{*}$ and $w$ are defined as follows: The value $w$ is a tension parameter influencing how closely the resulting surface follows the initial net. For $w=0$, e happens to be the midpoint of the edge $\overline{\mathbf{p}_{0} \mathbf{q}_{0}}$. In order to guarantee the smoothness of the limiting surface the value $w$ should be in the interval $(0, \sqrt{5}-1]$. Best results are obtained by choosing $w$ close to the standard 


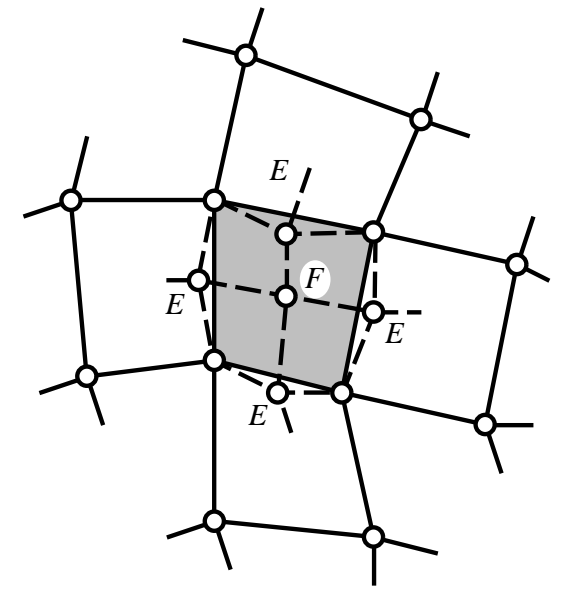

Figure 2: Splitting a quadrilateral face by $E$ vertices and $F$-vertices.

value $w=1$ [DGL87]. The point $\mathbf{q}^{*}$ is given by

$$
\begin{aligned}
\mathbf{q}^{*}:=\left(\frac{4}{n} \sum_{i=0}^{n-1} \mathbf{p}_{i}-\left(\mathbf{p}_{n-1}+\mathbf{p}_{0}+\mathbf{p}_{1}\right)\right. \\
+\frac{w}{4+w}\left(\mathbf{k}_{n-2}+\mathbf{k}_{n-1}+\mathbf{k}_{0}+\mathbf{k}_{1}\right) \\
\left.-\frac{4 w}{(4+w) n} \sum_{i=0}^{n-1} \mathbf{k}_{i}\right)
\end{aligned}
$$

and the point $\mathbf{p}^{*}$ is defined analogously. Here, $n$ denotes the valence of the vertex $\mathbf{q}_{0}$. In the regular case, $n=4$, the formula reduces to $\mathbf{q}^{*}=\mathbf{p}_{2}$. Since the new vertices which are inserted during the refinement process always have valence 4 , the general formula (2) is only needed to subdivide the edges directly emanating from a singular vertex (valence $\neq 4$ ). In the regular regions which cover most of the net, no further computation is necessary (cf. Fig. 4).

A face-point $\mathbf{f}$ is computed by applying (1) to four succeeding edge-points:

$$
\mathbf{f}:=\frac{4+w}{8}(\mathbf{b}+\mathbf{c})-\frac{w}{8}(\mathbf{a}+\mathbf{d}) \text {. }
$$

There are two possibilities for choosing this set of edge-points a, b, c, and $\mathbf{d}$. However, rule (2) is especially designed to make both alternatives equivalent. Hence, it does not matter in which direction rule (3) is applied since both lead to the same result.

If a refinement scheme is to be used in practical modeling or reconstruction applications, it must provide features that allow the definition of creases and cusps [Hop94]. These requirements

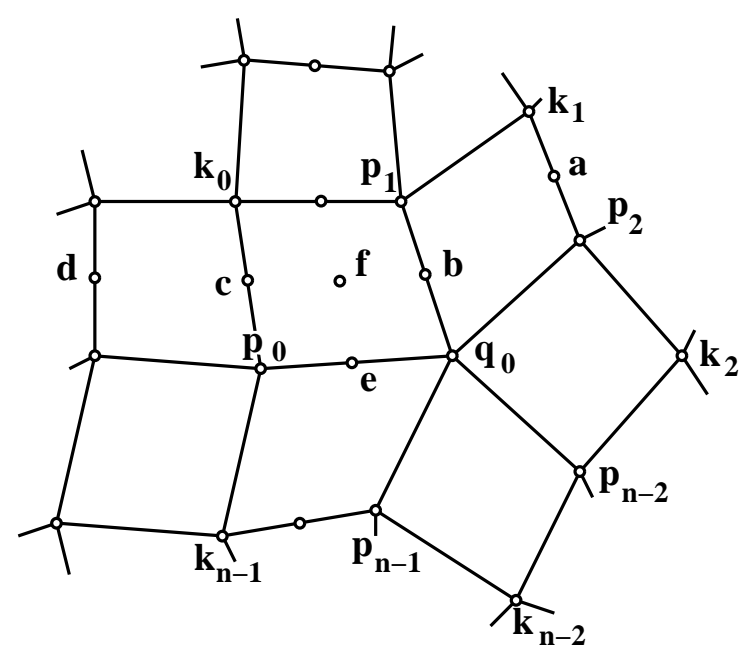

Figure 3: Labeling the vertices in the vicinity of the edge $\overline{\mathbf{p}_{0} \mathbf{q}_{0}}$.

can be satisfied if the scheme includes special rules for the refinement of open nets which yield well-behaved boundary curves that interpolate the boundary polygons of the given net. Having such a scheme, creases can be modeled by joining two separate subdivision surfaces along a common boundary curve and cusps result from a topological hole in the initial net which geometrically shrinks to a single point (cf. Fig. 5).

In order to refine open nets, we have to define additional rules for refining edges on or near the boundary. $C^{0}$-joins of subdivision surfaces whose initial nets have a common boundary polygon, require that the rules for the subdivision of boundary edges does not depend on inner vertices. Hence, we compute new edge vertices on the boundary by applying rule (3) where in this case $\mathbf{a}, \mathbf{b}, \mathbf{c}$ and $\mathbf{d}$ represent four neighboring boundary vertices.

In cases where three or more subdivision surfaces meet at a common point $\mathbf{c}$, it is necessary to allow piecewise smooth boundary curves. Let $\mathbf{a}, \mathbf{b}, \mathbf{c}$, and $\mathbf{d}$ be four neighboring boundary vertices of one of the nets. Since c belongs to more than three adjacent surfaces, it has to be a breakpoint in the piecewise smooth boundary curves. We define the corresponding refinement rule by replacing $\mathbf{d}$ by $\mathbf{d}^{*}:=2 \mathbf{c}-\mathbf{b}$ in (3).

Well-defined rules for the subdivision of inner edges emanating from a boundary vertex can be obtained by linearly extrapolating the boundary faces. This gives an additional layer of faces at the boundaries of the net, making former bound- 

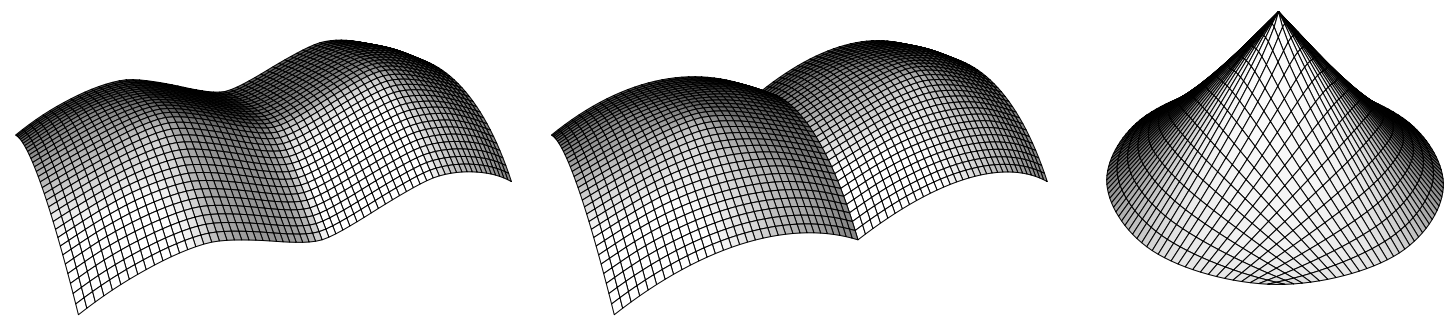

Figure 5: Modeling sharp features (piecewise smooth boundary, crease, cusp)
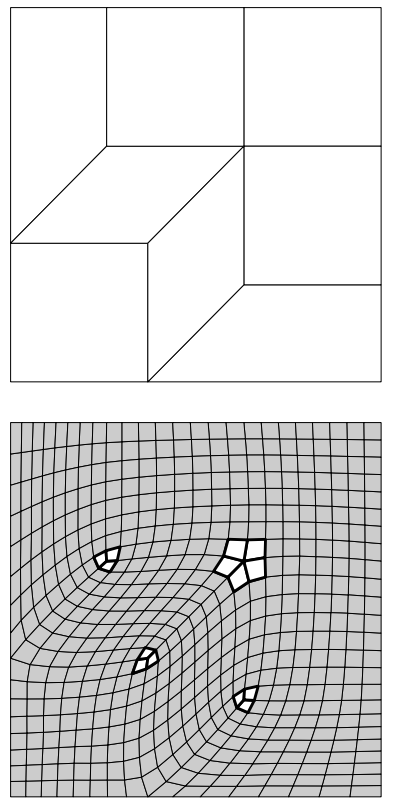

Figure 4: Singularities in the refined net.

\section{Convergence Analysis}

The refinement scheme defined in the last section belongs to the class of stationary subdivision schemes [Dyn91] since the same formulas are used on every refinement level. In the vicinity of any vertex, such schemes can be described in terms of a matrix which maps vertices from the $m$ th level to the $(m+1)$ st level. In [Rei95, Pra95] sufficient conditions on the eigen structure of this matrix are derived which guarantee the convergence of the sequence of nets to a tangent-plane continuous limiting surface.

These condition are that the leading eigen values have to be $\lambda_{1}=1$ and $\lambda_{2}=\lambda_{3}<1$ and the eigen vectors corresponding to $\lambda_{2}$ and $\lambda_{3}$ allow the definition of a regular paramerization with respect to which $C^{1}$-continuity is achieved [Rei95]. We omit the details of the straightforward verification of these criteria and refer to [Kob96].

\section{Approximation Order}

rules for inner edges can be applied. More precisely, if $\mathbf{q}_{1}, \ldots, \mathbf{q}_{n}$ are the inner vertices connected to the boundary vertex $\mathbf{p}$ then we can extrapolate by $\mathbf{p}^{*}:=2 \mathbf{p}-\frac{1}{n} \sum_{i} \mathbf{q}_{i}$.

In the case of a boundary face $\{\mathbf{p}, \mathbf{q}, \mathbf{r}, \mathbf{s}\}$ where the boundary vertex $\mathbf{p}$ is not connected to any inner vertex we have to distinguish two constellations. If $\mathbf{p}$ is a breakpoint in the piecewise smooth boundary curve, we add the extrapolated face $\left\{\mathbf{p}, \mathbf{q}^{*}, \mathbf{r}^{*}, \mathbf{s}^{*}\right\}$ with $\mathbf{q}^{*}:=2 \mathbf{p}-\mathbf{q}$, $\mathbf{s}^{*}:=2 \mathbf{p}-\mathbf{s}$ and $\mathbf{r}^{*}=\mathbf{q}^{*}+\mathbf{s}^{*}-\mathbf{p}$. If $\mathbf{p}$ is no breakpoint, we add the vertex $\mathbf{p}^{*}:=2 \mathbf{p}-\mathbf{r}$.

Connecting the extrapolated vertices corresponding to neighboring boundary vertices gives the additional layer of extrapolated faces. After the refinement, these faces have to be deleted.
The interpolation scheme presented in this paper is designed to generate free form surfaces. Since we do not exploit any meta-knowlegde about the geometry but only the information given by the initial net, we cannot expect to exactly reproduce basic shapes like spheres or cylinders. However, this lack is tolerable if we can guarantee that denser information about the considered object, i.e., a finer initial net with correct vertex positions (on the surface to be approximated), significantly improves the quality of the approximation.

From [DGL87] we know that, on regular tensor product meshes, the above presented interpolation scheme reproduces the polynomials $1, x, y$, and $x y$. Further, if we restrict the tension parameter to $w=1$, the scheme even reproduces 
all polynomials up to bi-degree 3 . For irregular meshes, we have to apply the rule (2) in its full generality. Since the scheme generates $C^{1}$-surfaces in the limit, we can use the special parametrization induced by the eigen structure of the subdivision matrix with respect to which the refinement scheme has at least linear precision.

Keeping in mind that the position of the new points only depends on a fixed number of neighboring vertices, it is obvious that for sufficiently smooth surfaces, the approximation order directly follows form the polynomial precision of the scheme. This can be seen by looking at a local Taylor expansion of the surface. Hence, we obtain an approximation order of $O\left(h^{2}\right)$ which increases to $O\left(h^{4}\right)$ over regular regions of the net if the tension parameter is set to $w=1$. Here, $h$ denotes any uniform measure of the grid size, e.g., average or maximal length of edges in the initial mesh. In order to achieve the best approximation, let $w:=1$ for the rest of this section.

Obviously, the $O\left(h^{2}\right)$-term in the vicinity of extraordinary vertices asymptotically dominates the error functional such that in general, we only get quadratic convergence. However, if we can ensure that the number of singularities in the initial net does increase significantly with the density of the input data then we achieve the full $O\left(h^{4}\right)$ convergence. The assumption that the number of singular vertices in a mesh $P_{h}$ approximating a surface $S$ can be bounded by a constant is quite realistic since the amount of singularities somewhat reflects the topological or geometrical complexity of the shape of $S$ which is independent of $h$.

Suppose $S: \Omega \rightarrow \mathbb{R}^{3}$ is a parametrization of the surface and $P_{h}$ is an interpolating quadrilateral mesh whose average meshsize is measured by the stepwidth $h$ (in the domain $\Omega$ ). The size of the local region around singular vertices where $O\left(h^{2}\right)$-convergence occurs, is determined by topological distance on $P_{h}$, i.e., by the minimal number of edges connecting some vertex to a singular one. Hence the corresponding portion of the domain $\bar{\Omega} \subset \Omega$ covers an area of $\|\bar{\Omega}\|=O\left(h^{2}\right)$ if the number of singular vertices is finite. Under these conditions the error functional can be written as

$$
\begin{aligned}
E_{h}(S) & =\int_{\Omega \backslash \bar{\Omega}} O\left(h^{4}\right)+\int_{\bar{\Omega}} O\left(h^{2}\right) \\
& =O\left(h^{4}\right) .
\end{aligned}
$$

Several algorithms to reduce the number of singular vertices in a mesh have been proposed [Eck95, Sch95]. The meshes one encounters in practical applications usually have a constant or very slowly increasing number of singularities as the mesh-size $h$ is reduced, e.g., 3D-scanners often measure an object along parallel profiles or along radial meridians and thus generate tensorproduct-type data.

\section{Examples}

We use the refinement scheme to approximate two test surfaces: the unit-sphere and the graph of the function $\cos \left(\sqrt{x^{2}+y^{2}}\right)$ over the domain $\Omega=[0,1]^{2}$. In the sphere case the number of singular vertices is constant, since the initial nets are generated by using nets as depicted in Fig. 1 and projecting the vertices onto the sphere. The graph-surface is approximated by taking equidistant samples in $x$ - and $y$-direction.

\begin{tabular}{|l|l|c|c|c|}
\hline$h$ & $\int_{\Omega}|\varepsilon|$ & $q_{1}$ & $\max |\varepsilon|$ & $q_{\infty}$ \\
\hline \hline 1 & $3.11 \mathrm{e}-04$ & & $5.64 \mathrm{e}-04$ & \\
\hline .5 & $1.95 \mathrm{e}-05$ & 15.9 & $3.59 \mathrm{e}-05$ & 15.7 \\
\hline .25 & $1.22 \mathrm{e}-06$ & 16.0 & $2.26 \mathrm{e}-06$ & 15.9 \\
\hline .125 & $7.57 \mathrm{e}-08$ & 16.1 & $1.41 \mathrm{e}-07$ & 16.0 \\
\hline
\end{tabular}

Table 1: Approximation errors for the graph of $\cos (r), r^{2}=x^{2}+y^{2}$ over $\Omega=[0,1]^{2}$, stepwidth $h$. We show the errors with respect to the $L_{1^{-}}$and the $L_{\infty}$-norms. The $q$-columns contain the ratio of successive errors indicating $O\left(h^{4}\right)$ convergence.

In order to demonstrate the different rates of convergence in the parametric case, we compute the approximation error separately for the regular portions of the net. In the functional settings this distinction is not necessary since the nets have no extraordinary vertices.

\section{Numerical Experiments}

In order to evaluate the practical usefulness of the presented scheme, we apply it to a set of examples and compare our results to those obtained by standard techniques based on implicit representations used, e.g., in the context of adaptive finite element analysis by [SR91, Bau95]. For both types of representation we 


\begin{tabular}{|l|l|l|l|c|}
\hline$h$ & $\int_{\text {reg }}|\varepsilon|$ & $q_{\text {reg }}$ & $\int_{\text {tot }}|\varepsilon|$ & $q_{\text {tot }}$ \\
\hline \hline .25 & $1.64 \mathrm{e}-03$ & & $5.36 \mathrm{e}-03$ & \\
\hline .125 & $1.22 \mathrm{e}-04$ & 13.5 & $4.02 \mathrm{e}-04$ & 13.3 \\
\hline .0625 & $8.09 \mathrm{e}-06$ & 15.1 & $3.12 \mathrm{e}-05$ & 12.9 \\
\hline .03125 & $4.88 \mathrm{e}-07$ & 16.6 & $2.46 \mathrm{e}-06$ & 12.7 \\
\hline
\end{tabular}

Table 2: Approximation errors for the sphere. The errors are normalized to the volume of the sphere. The stepwidth $h=1$ corresponds to a cube as initial approximation.

compare approximations obtained by adaptive uniform refinement and adaptive irregular refinement [Bau95]. Both schemes are also tested in combination with mesh smoothing as suggested by [Sch93, Sch95, Ric96], where the element shape is optimized after each refinement step by using a local smoothing technique. We use the following abbreviations:

- unif ... uniform refinement, exact geometry

- adap ... non-uniform refinement, exact geometry

- smoo ... with mesh smoothing

- orig ... without mesh smoothing

- inte ... mesh generation by the proposed refinement scheme

\section{Cylindrical Shell with Hole}

The cylindrical shell, originally proposed by [Sco69] is rigidly supported at both curved boundaries (cf. Fig. 6 ) and loaded by a uniformly distributed load in vertical direction. The vertical displacement $v$ at the center of the free edges is the quantity compared in the analysis.

Due to symmetry, only a quarter of the shell has to be discretized in the finite element model. The starting meshes used in the analysis are shown in Fig. 7. They consist of 32 elements and 45 nodes. In order to achieve a sufficiently good approximation of the geometry, 5 nodes are chosen in the latitudinal direction. Using an initial mesh having 8 elements ( 15 nodes), it turns out that the geometry is not well approximated and the numerical analysis results in a displacement being $15 \%$ larger than the exact value.

The mesh given in Fig. $7 \mathrm{~b}$ is a slight modification of the mesh in Fig. 7a, which results from

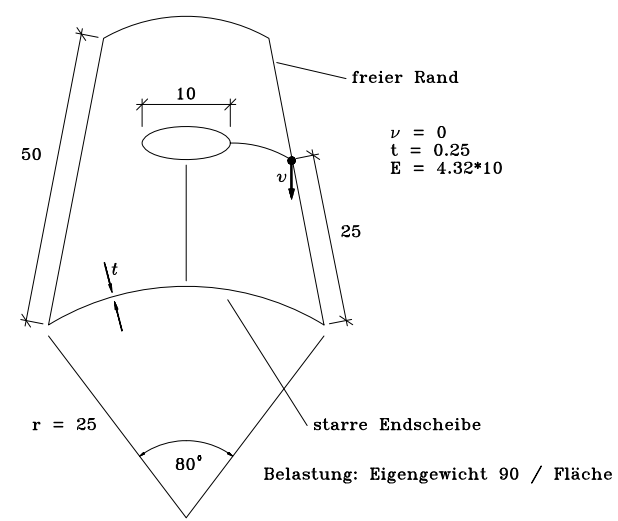

Figure 6: Circular shell with hole, geometry, material properties, and loading

mesh smoothing. As the element boundaries are no longer aligned with the direction of the main curvatures, this leads to a significantly different initial geometry for the interpolation algorithm. The modification of the mesh leads to displacements differing about $10 \%$ from the correct values.

In Fig. 8 the meshes for uniform and irregular adaptive refinement based on the interpolatory refinement scheme are given. Convergence diagrams are depicted for the displacement $v$ in Fig. 9 and for the relative error in energy in Fig. 10. For comparison, the curves for other refinement strategies which are performed on the implicit representation of the exact geometry, are also included.

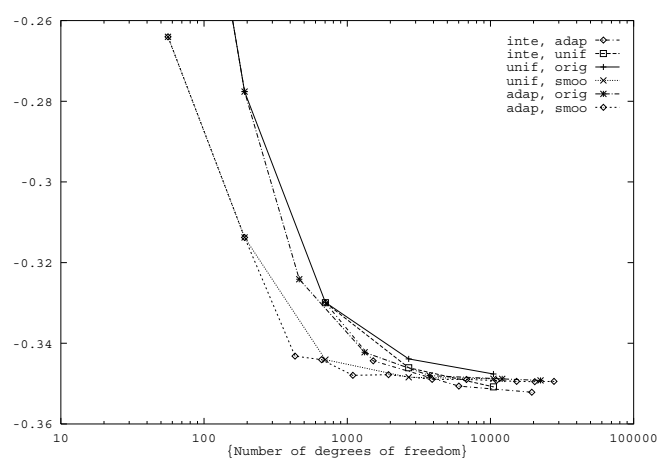

Figure 9: Circular shell with hole, Convergence of midside displacement

\section{Discussion}

If the starting mesh is reasonably fine, the interpolation algorithm leads to almost the same results as the refinement on the implicitly given 

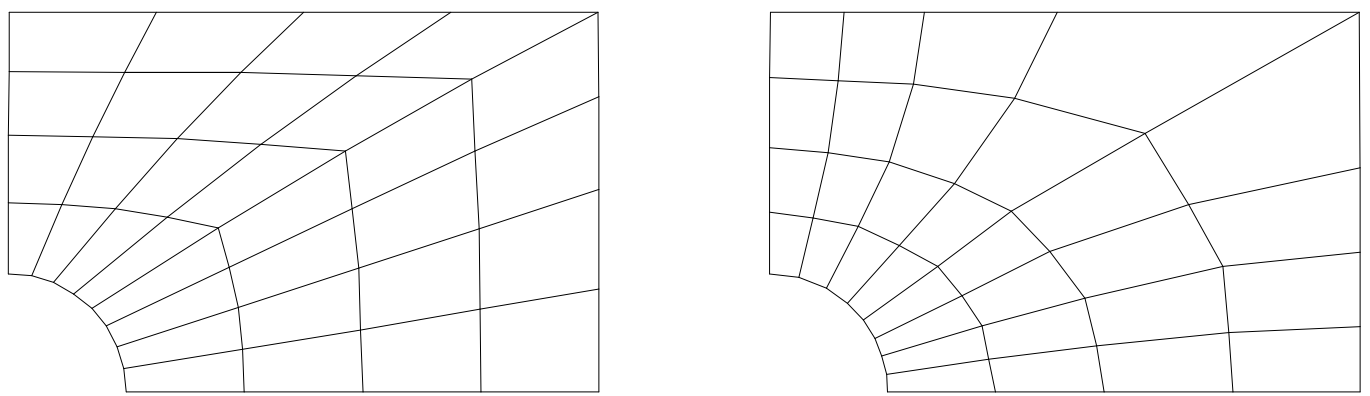

Figure 7: Circular shell with hole, a) basic, b) smoothed starting mesh
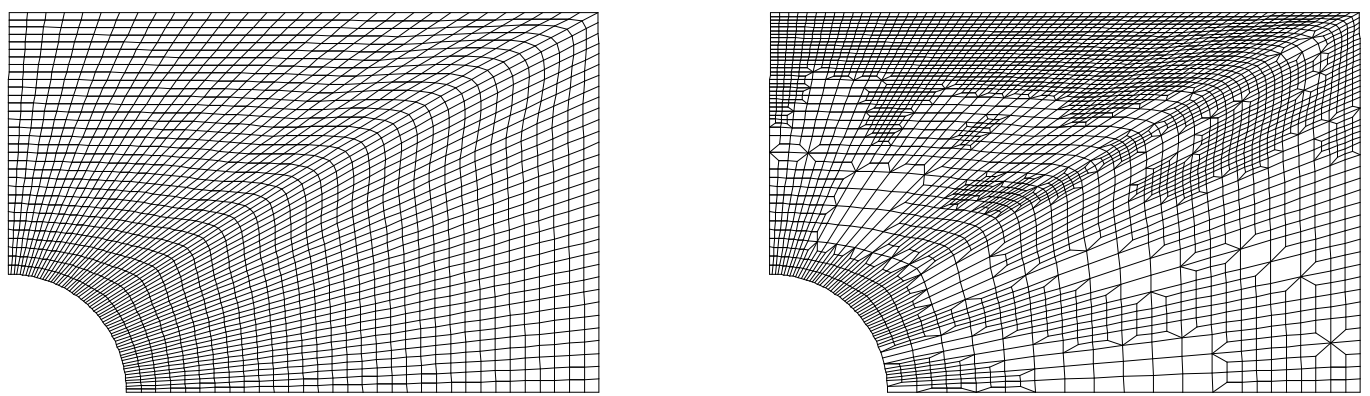

Figure 8: Circular shell with hole, a) uniform, b) adaptive irregular final mesh

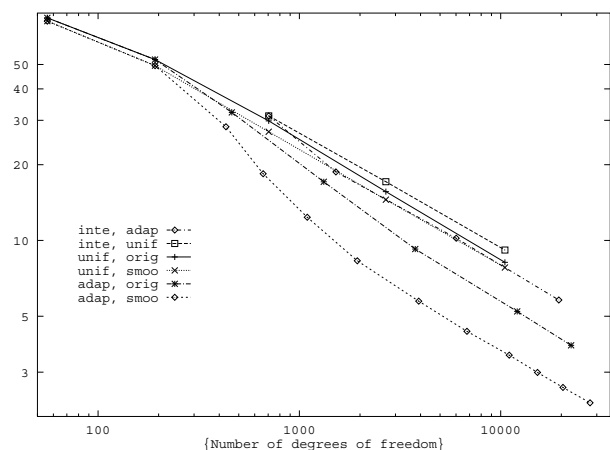

Figure 10: Circular shell with hole, Convergence of energy error

geometry for uniform refinement. For adaptive irregular refinement the results can be worse if the starting mesh is too coarse because in this case the geometry itself is not approximated correctly. According to the observations in the last section, the approximation order of the refinement scheme guarantees that a higher density of the initial mesh strongly improves the quality of the approximation of the geometry.

A combination of the refinement scheme with smoothing techniques [Sch93] is not possible, as the interpolation nodes are fixed in the current form of the interpolation algorithm. However, the convergence of the displacements is very good - only the algorithms which use smoothing techniques lead to better results. It turns out that the algorithm is well suited for practical engineering applications.

\section{Composite Cylinder-Plate structure}

The second example is the intersection of a cylinder and a plate by an angle of 45 degrees. The width of the quadratic plate is $w=15$ and the cylinder of radius $r=6$ has an average length of $l=10$. The thickness of the cylinder and of the plate are both $t=1$. The modulus of Elasticity is $E=10^{6}$ and the Poisson ratio $\nu$ is set to zero. The plate is simply supported. A uniformly distributed load is applied at the free edge of the cylinder and is directed along the axis of the cylinder.

As we explained earlier, the crease along the intersection curve can be modeled by joining two separate parts, the cylinder and the the plate, having a common boundary polygon. Hence, common nodes have to be stored twice since otherwise the interpolatory refinement would generate a smooth join. 


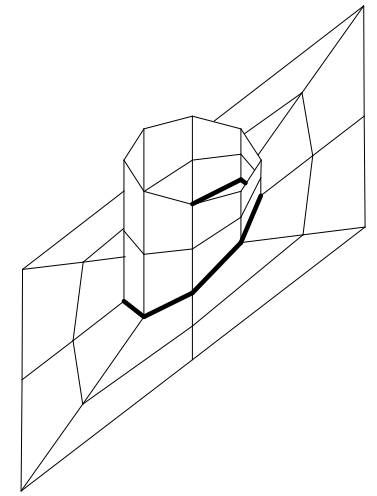

Figure 11: Composite structure (common boundary indicated), starting mesh

The initial and the final meshes of the adaptive analysis are shown in Fig. 11 and 12. For an exact interpolation of the plate it is sufficient to define 4 corner vertices (linear precision). The cylinder is defined by the boundary polygons at the free edge and at the intersection. Using 8 nodes along these boundaries guarantees a sufficiently accurate reproduction.

\section{Discussion}

The convergence diagram for the energy error in Fig. 13 shows a slightly reduced convergence for the interpolation algorithm in combination with uniform refinement compared to the results obtained by using the other algorithms. With adaptive irregular refinement the results obtained with our scheme are almost identical to all other results.

\section{Conclusion}

The presented scheme to generate adaptively refined quadrilateral meshes allows to approximate arbitrary surfaces with holes and sharp local features. The quality of the approximation is sufficiently well, even if the scheme is applied to moderately detailed initial meshes. Due to the symmetry of the refinement rules for the edges around a vertex, the local distortion is reduced, i.e., if $n$ edges meet at a common vertex, the angles between them tend to $\frac{2 \pi}{n}$. This regularization effect causes the shapes of adjacent faces to become alike.

The slightly less accurate results obtained by using the interpolatory refinement scheme instead of an exact representation of the given objects (cf. last Section) stems from the fact that the subdivision scheme computes new vertices without any meta-knowledge about the shape (in this case that the surfaces are cylindrical). However, the scheme is mainly designed for computations on free form surfaces of which only discrete points are given and an exact representation is not known. Therefore also other approaches based on splines would have to guess the actual shape.

The power of the scheme is demonstrated best by applying it to a more sophisticated model. The surface in Fig. 14 is generated by using the coarse approximation as input data and refining twice. No additional information is necessary. Constructing a piecewise smooth spline surface interpolating this irregular net would be rather complicated.

The major advantage of this scheme compared to other approaches therefore is its striking simplicity. Only local affine combinations of points have to be computed in order to refine a given net. Almost no special cases concerning the local topology of the mesh have to be considered. The examples have been generated by our implementation which provides all the presented features including adaptive irregular refinement with $C^{0}$-consistency preservation. (please contact: kobbelt@cs.wisc.edu)

\section{References}

[BCX94a] C. Bajaj / J. Chen / G. Xu, Modeling with Cubic A-Patches, ACM Trans. Graph. 1994

[BCX94b] C. Bajaj / J. Chen / G. Xu, Smooth Low Degree Approximations of Polyhedra, preprint 1994

[BD86] K. Bathe / E. Dvorkin, A formulation fo General Shell Elements - The Use of Mixed Interpolation of Tensorial Components, Int. J. Num. Meth. Eng. 22(1986)

[Bau95] M. Baumann / K. Schweizerhof, Adaptive mesh generation on arbitrarily curved shell structures, in M. Papadrakakis and B. Topping, eds, Proceedings of 2nd Int.Conf. on Comput. Structures Techn. in 

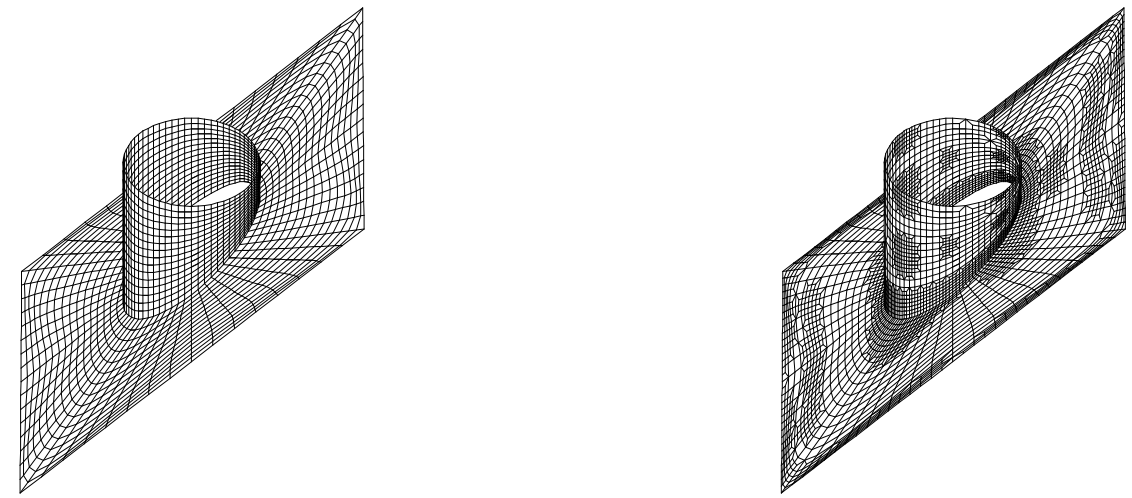

Figure 12: Composite structure, final meshes a) uniform b) irregular refinement

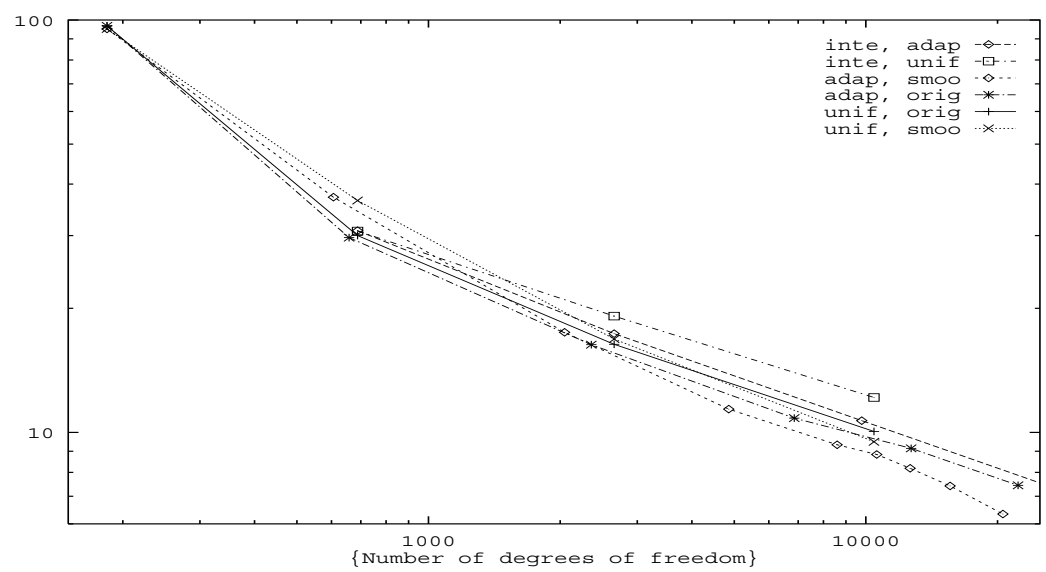

Figure 13: Composite structure, convergence diagram for energy error

"'Advances in Finite Element Techniques", Civil-Comp Press Edinburgh, 1995.

[CC78] E. Catmull / J. Clark, Recursively Generated B-Spline Surfaces on Arbitrary Topological Meshes, CAD 10 (1978), pp. 350-355

[DGL87] N. Dyn / J. Gregory / D. Levin, A 4-Point Interpolatory Subdivision Scheme for Curve Design, CAGD 4(1987), pp. 257-268

[DGL90] N. Dyn / J. Gregory / D. Levin, A Butterfly Subdivision Scheme for Surface Interpolation with Tension Controll, ACM Trans.Graph. 9 (1990), pp. 160-169

[DS78] D. Doo / M. Sabin, Behaviour of Recursive Division Surfaces Near Extraordinary Points, CAD 10 (1978), pp. $356-360$
[Dyn91] N. Dyn, Subdivision Schemes in Computer Aided Geometric Design, Advances in Numerical Analysis II, Wavelets, Subdivisions and Radial Functions, W.A. Light ed., Oxford University Press, 1991, pp. 36-104.

[Eck95] Eck / DeRose / Duchamp / Hoppe / Lounsbery / Stuetzle, Multiresolution Analysis of Arbitrary Meshes, Report, University of Washington, UW-CSE-95-01-02

[Hop94] H. Hoppe, Surface Reconstruction from unorganized points, Thesis, University of Washington, 1994

[HL93] J. Hoschek / D. Lasser, Fundamentals of computer-aided geometric design, A.K. Peters, 1993

[Kob95] L. Kobbelt, Iterative Erzeugung glatter Interpolanten, Thesis, Universität Karlsruhe, 1995 

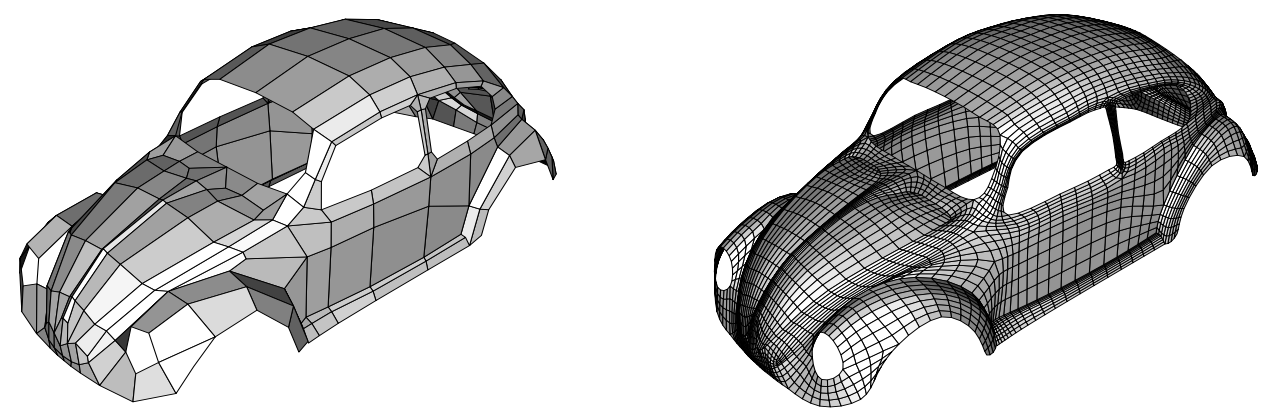

Figure 14: Generating complex shapes by interpolatory subdivision

[Kob96] L. Kobbelt, Interpolatory Subdivision on Open Quadrilateral Nets with Arbitrary Topology, to appear in Computer Graphics Forum

[Loo87] C. Loop, Smooth Subdivision for Surfaces Based on Triangles, Master Thesis, University of Utah, 1987

[Loo94] C. Loop, $A G^{1}$ triangular spline surface of arbitrary topological type, CAGD 11 (1994), pp. 303-330

[Pra95] H. Prautzsch, $C^{k}$-analysis of subdivision surfaces at extraordinary points, submitted, 1995

[PSB90] L. Plank / E. Stein / D. Bischoff, Accuracy and Adaptivity in the Numerical Analysis of Thin Walled Structures, Comp. Meth. in Appl. Mech. Eng., 82 (1990)

[Rei95] U. Reif, A unified approach to subdivision algorithms near extraordinary vertices, CAGD 12 (1995), pp. 153174

[Ric96] J. Riccius / K. Schweizerhof / M. Baumann, Combination of adaptivity and mesh smoothing for the finite element analysis of shells with intersections, to appear in Int. j. numer. methods eng.

[SF89a] J. Simo / D. Fox, On a Stress Resultant Geometrically Exact Shell Model. Part I: Formulation and Optimal Parametrization, Comp. Meth. Appl. Mech. Eng. 72 (1989)

[SF89b] J. Simo / D. Fox, On a Stress Resultant Geometrically Exact Shell
Model. Part II: The Linear Theory: Computational Aspects, Comp. Meth. Appl. Mech. Eng. 73 (1989)

[Sch93] K. Schweizerhof / J. Riccius / M. Baumann, Verbesserung von Finite Element Berechnungen durch Adaptivität und Netzglättung am Beispiel ebener und gekrümmter Fl"achentragwerke, Technical report, Universität Karlsruhe , Institut für Wissenschaftliches Rechnen und Mathematische Modellbildung, 1993

[Sch95] R. Schreiber, Kombinatorisch optimierte Konstruktion fast-regulrer Polygonnetze Thesis, Universität Stuttgart, 1995

[Sco69] A. Scordelis / K. Lo, Computer analysis of cylindrical shells, J. of American Concrete Institute, 61 (1996), $539-561$

[SR91] E. Stein / W. Rust, Mesh adaptations for linear $2 D$ finite-element discretizations in structural mechanics, especially in thin shell analysis, J. Comput. Appl. Math., 36 (1991), 107-129

[ZSS96] D. Zorin / P. Schroeder / W. Sweldens, Interpolating Subdivision for Meshes of Arbitrary Topology to appear in SIGGRAPH Proceedings 1996 